\title{
Tiroiditis por trauma en cuello luego de intento de suicidio. Reporte de caso y revisión de la literatura
}

\author{
Carlos Alfonso Builes Barrera ${ }^{1}$, Juan David Gomez ${ }^{1}$, Alejandro Román González ${ }^{1}$
}

\author{
${ }^{1}$ Internista, Endocrinólogo. Grupo Endocrinología y Metabolismo, \\ Universidad de Antioquia ( $U$ de A), Hospital Universitario San \\ Vicente Fundación. Medellín, Colombia. \\ Correspondencia: \\ Carlos Alfonso Builes Barrera, MD \\ Correo electrónico: endocrinobuiles@gmail.com \\ Conflicto de interés: ninguno
}

\section{Resumen}

La tiroiditis es un fenómeno inflamatorio de la tiroides, de causas diversas, incluyendo raramente traumas de la región anterior del cuello. Se presenta el caso de un paciente con depresión mayor de curso crónico, con síntomas sicóticos y farmacodependencia, quien intenta suicidarse mediante ahorcamiento. Ingresa con un Glasgow de 3/15, por lo cual requiere intubación orotraqueal inmediata, posterior traslado a unidad de cuidados intensivos y ventilación mecánica durante diez días. Durante este periodo desarrolla taquicardia persistente, diaforesis y alteraciones del sensorio, se encontró TSH en $0,00(0,4-$ $4 \mathrm{mUI} / \mathrm{ml})$; una T3 total en $2,42,(0,8-2,0 \mathrm{ng} / \mathrm{ml})$ y T4 libre $>6$ $(0,93-1,70 \mathrm{ng} / \mathrm{dL})$, la gammagrafía de tiroides con tecnecio 99 mostró bloqueo de la captación. Se sospechó tormenta tiroidea y recibió propranolol 80 mg vía oral cada 8 horas en forma continua e hidrocortisona $50 \mathrm{mg}$ intravenosa cada 8 horas durante 5 días. Los controles, dos semanas después, muestran TSH en 0,00 (0,4-4 uUI/ml) y una T4 libre de 1,78 (0,93-1,70 ng/dl) y cuatro días después normalización de la T4 libre (1,45 ng/dl), acompañado de mejoría clínica de los síntomas adrenérgicos.

Se presenta el caso de un paciente con tiroiditis postrauma, entidad que debe ser sospechada en pacientes con trauma en el cuello que presenten síntomas de tirotoxicosis.

Palabras clave: Tiroiditis, estrangulación, trauma, suicidio, gammagrafía

\section{Abstract}

Thyroiditis is an inflammatory disorder of the thyroid, which stems from a variety of causes including in some rare cases trauma to the neck. We present the case of a male patient with chronic depression associated with psychotic symptoms and drug abuse that tried to commit suicide through hanging. The patient had a
Glasgow Coma Scale 3/15 requiring orotracheal intubation, a stay in the intensive care unit and mechanical ventilation for 10 days. While in the ICU the patient developed persistent tachycardia, diaphoresis and changes in mental status. A suppressed TSH was found with a level of 0,00 (0,4-4 uUI/ml) and high thyroid hormone level (total $T_{3} 2,42$, (normal value 0,8-2,0 $\mathrm{ng} / \mathrm{ml}$ ) and free $T_{4}>6$ (normal value 0,93-1,70 ng/dL). A technetium-99 thyroid scintygraphy showed blocked uptake. With those findings, a thyroid storm was suspected and management was begun with propranolol 80 $\mathrm{mg}$ per mouth every 8 hours and IV hydrocortisone $50 \mathrm{mg}$ every 8 hours for 5 days. Two weeks later, the TSH was 0,00 (0,4-4 uUI) $\mathrm{ml}$ ) and the free $T_{4}$ was 1,78 (normal value 0,93-1,70 $\mathrm{ng} / \mathrm{dl}$ ). Four days later, the free 74 concentration was normal $(1,45 \mathrm{ng} / \mathrm{dl})$ with a clinical improvement of the adrenergic symptoms.

In conclusion, thyroiditis should be suspected in patients with neck trauma with compatible clinical manifestations.

Key words: Thyroiditis, strangulation, trauma, suicide, thyroid scan.

\section{Introducción}

La glándula tiroides almacena en su interior hormonas tiroideas preformadas, cuya liberación masiva durante procesos inflamatorios genera síntomas de tirotoxicosis ${ }^{(1)}$. La tiroiditis subaguda tiene múltiples etiologías, incluyendo irradiación, consumo de litio, amiodarona, fenómenos autoinmunes contra la tiroides, consumo de carne contaminada con glándula tiroides, ingestión intencionada o accidental de hormona tiroidea y trauma ${ }^{(1)}$. Diversas formas de trauma se han descrito como causales de tiroiditis, incluyendo trauma por artes marciales $^{(2)}$, manipulación en procedimientos quirúrgicos ${ }^{(3-6)}$, trauma con cinturón de seguridad de vehículo ${ }^{(7)}$, biopsia de tiroides $^{(8,9)}$, examen físico vigoroso ${ }^{(10)}$ e incluso casos de tormenta tiroidea por estrangulación ${ }^{(11)}$.

En el presente artículo se reporta el caso de un paciente que desarrolla una tiroiditis subaguda luego de intento de suicidio mediante ahorcamiento.

\section{Reporte de caso}

Hombre de 24 años de edad, llevado e ingresado al servicio de urgencias luego de intento de ahorcamiento con un lazo. 
Como antecedente patológico tenía síntomas depresivos de seis años de evolución, un intento de suicidio previo y abuso de alcohol y marihuana.

Al examen físico de ingreso se encontró un Glasgow 3/15 con pupilas isocóricas, normorreactivas a la luz, frecuencia cardiaca (FC) de 100 latidos por minuto (lpm), 20 respiraciones por minuto (rpm), presión arterial (PA) de 89/69 mmHg. Con laceración superficial de la piel del cuello, en tercio superior del lado izquierdo. Se realizó intubación orotraqueal para protección de la vía aérea, se descartó luxación de vértebras cervicales, compresión medular y lesión cerebral mediante TAC de cráneo.

El paciente requirió ventilación mecánica en UCI durante 10 días, allí presentó fiebre y se documentó neumonía por aspiración con aislamiento de Staphylococcus aureus sensible a meticilina y Burkholderia cepacia, para lo cual recibió tratamiento antibiótico.

El décimo día de hospitalización presentó taquicardia en reposo FC de 128 lpm, PA 120/60 mm/Hg, una FR de 25 rpm, diaforesis, palidez mucocutánea, ansiedad, agitación psicomotora y aumento generalizado y simétrico de los reflejos osteotendinosos con clonus aquiliano. En su evaluación por una sospecha de delirium (día 13 de hospitalización) se encontró TSH suprimida: 0,00, (valor de referencia VR: 0,4-4 mUI/ml); T3 total elevada: 2,42, (VR: 0,8-2,0 ng/ml) y una T4 libre elevada: > 6, (VR: 0,93-1,70 ng/dL). Ante la sospecha de tormenta tiroidea, se ordenó iniciar metimazol, (pero no lo recibió), hidrocortisona intravenosa y propranolol.

Es evaluado por endocrinología, encontrando un paciente, tranquilo, hipoactivo, con FC de $96 \mathrm{lpm}, \mathrm{PA}$ 116/50 mmHg, FR de 28 rpm, temperatura corporal 37,2, peso 55 kilogramos, mirada fija, bradicinético, bradipsíquico, sin respuesta verbal espontánea, ni con estímulo táctil. Obedeciendo órdenes motoras sencillas, movilizando las cuatro extremidades y con hiperreflexia generalizada. No presentaba exoftalmos, con tiroides palpable, de tamaño normal y con una laceración semicircular en la cara lateral izquierda del cuello (figura 1) y sin dermopatía pretibial. Se continúa sólo manejo con propranolol 80 mg cada 8 horas e hidrocortisona $50 \mathrm{mg}$ cada 8 horas durante 5 días y se realiza una gammagrafía con $3 \mathrm{mCI}$ IV de ${ }_{99 \mathrm{~m}} \mathrm{TC}$, con captación del radio fármaco severamente disminuida con tendencia al bloqueo y de distribución irregular (figura 2), apoyando el diagnóstico de tiroiditis. No se realizó ecografía de tiroides. Durante el tiempo de la hospitalización hubo disminución progresiva del nivel de T4 libre, sólo con uso de betabloqueador oral (tabla 1). Posteriormente, el paciente fue remitido a otra institución para continuar el manejo de su patología psiquiátrica de base. En resumen, se presenta el caso de un paciente con depresión mayor de curso crónico, con síntomas sicóticos, con farmacodependencia, quien intenta suicidarse mediante ahorcamiento, desarrollando como complicaciones neumonía por aspiración y tiroiditis por ahorcamiento.
Figura 1. Laceración en cuello. Laceración superficial de piel sobre el borde lateral izquierdo del cuello.

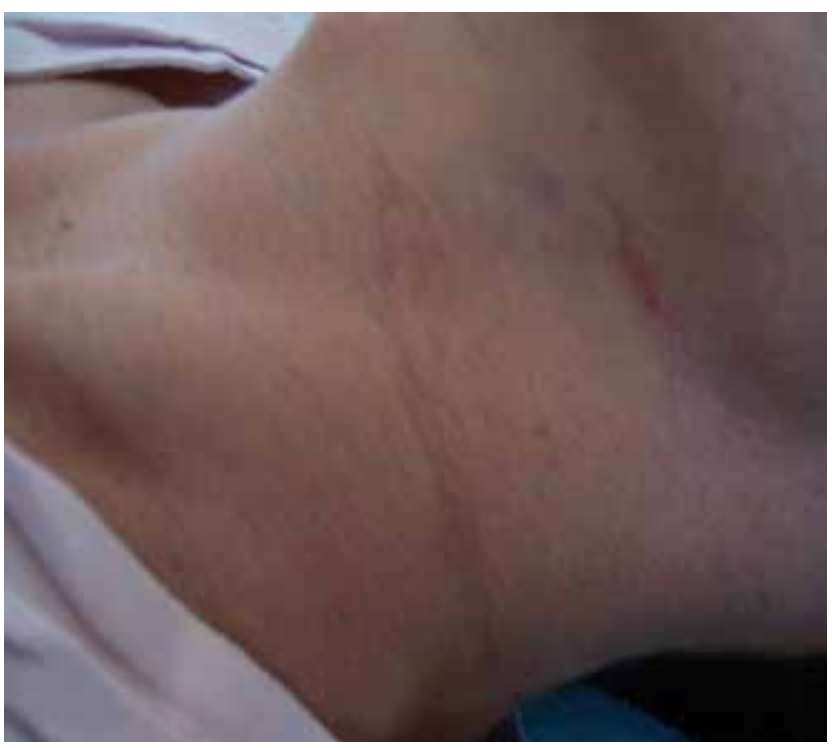

Figura 2. Gammagrafía de tiroides. Nótese el severo bloqueo de la captación del radiofármaco.

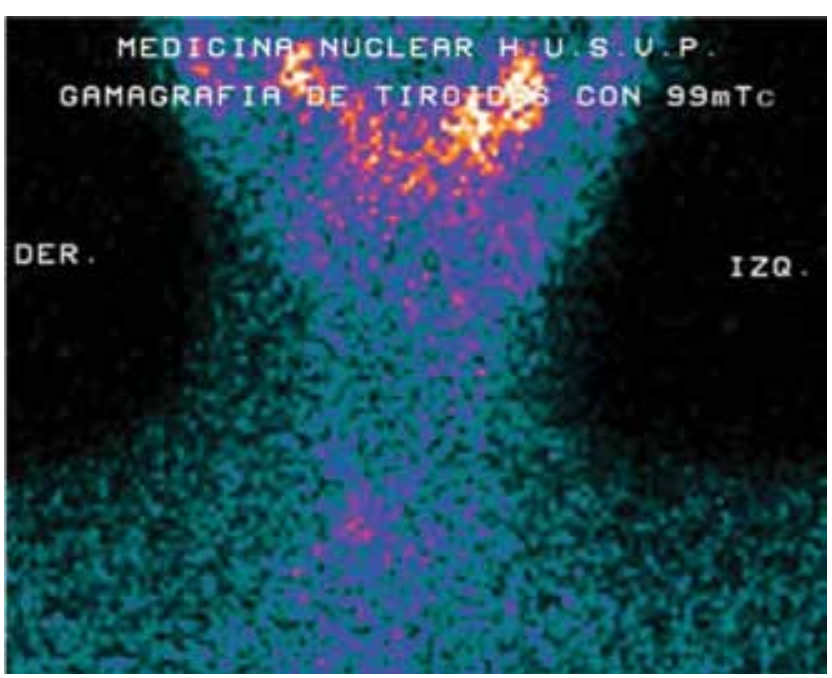

Tabla 1. Pruebas de función tiroidea durante la hospitalización.

\begin{tabular}{c|c|c|c|c}
\hline Examen & Día 13 & Día 27 & Día 31 & Valor de referencia \\
\hline $\mathrm{TSH}$ & 0,00 & 0,00 & & $0,4-4 \mathrm{mUl} / \mathrm{ml}$ \\
\hline $\mathrm{T}_{3}$ total & 2,42 & & & $0,8-2 \mathrm{ng} / \mathrm{ml}$ \\
\hline $\mathrm{T}_{4}$ libre & $>6$ & 1,78 & 1,45 & $0,93-1,7 \mathrm{ng} / \mathrm{dl}$ \\
\hline
\end{tabular}




\section{Discusión}

La tirotoxicosis no asociada a hipertiroidismo primario, desencadenada por tiroiditis subaguda, produce signos y síntomas variables que pueden ir desde una forma detectable únicamente en el laboratorio, hasta la forma grave de tormenta tiroidea. En el presente caso no se tiene evidencia de manifestaciones clínicas de la tríada clásica de la enfermedad de Graves-Basedow, ni de otras causas de tirotoxicosis. Las características clínicas, de laboratorio y de imaginología con bloqueo de la captación en la gammagrafía con ${ }^{99 \mathrm{~m}} \mathrm{Tc}$ soportan la evidencia de una tiroiditis postrauma del cuello causado por un lazo.

La consideración inicial de tormenta tiroidea se realizó por la presencia de fiebre, delirium y taquicardia en presencia de niveles aumentados de hormona tiroidea, con un puntaje de 50 en la escala de predicción clínica de tormenta tiroidea según Burch and Wartofsky ${ }^{(12)}$ (altamente probable si es mayor de 45). Se postula que no sólo es el valor de la hormona tiroidea, sino también el ascenso rápido del nivel circulante de hormona tiroidea luego del trauma el responsable del desarrollo de una tormenta tiroidea. El paciente recibió betabloqueador en dosis alta y esteroides para disminuir la conversión periférica de $\mathrm{T} 4$ a $\mathrm{T} 3$.

Ante la presencia del predominio de T4 más que T3, la rápida reducción de los niveles de $\mathrm{T} 4$ con sólo betabloqueadores y el bloqueo de la captación del ${ }^{99 \mathrm{~m}} \mathrm{Tc}$ se sustentó el diagnóstico de tiroiditis por trauma, motivo por el cual era innecesario el uso de metimazol, pues no hay un exceso de la síntesis de hormona tiroidea, sino una liberación masiva de hormona preformada, con una naturaleza transitoria. Aunque la ecografía tiroidea en patología difusa ha ganado terreno, no se tiene de rutina personal entrenado que permita diferenciar entre hipertiroidismo primario y tirotoxicosis transitoria por tiroiditis subaguda con base en el patrón de flujo Doppler, y se prefiere la realización de la gammagrafía de tiroides para aclarar, de una forma objetiva, el porcentaje de captación o atrapamiento y así definir tiroiditis subaguda ante la presencia de una marcada reducción de la captación ( ${ }^{131}$ ) o atrapamiento $\left({ }^{99 \mathrm{~m}} \mathrm{Tc}\right.$ ).

La mayor parte de la información de tiroiditis postraumática proviene de series pequeñas de casos (tabla 2), en las que se reporta supresión de la TSH y aumento en los niveles de las hormonas tiroideas circulantes, con síntomas leves de tirotoxicosis, en pacientes a quienes se realiza paratiroidectomía por hiperparatiroidismo primario/secundario o laringectomía por cáncer de laringe $\mathrm{e}^{(3-5,13-15)}$, esta elevación de las hormonas tiroideas circulantes tiene una naturaleza transitoria, con aumento de los niveles en la primera semana y normalización de los niveles de T4 y T3 entre 14 y 21 días, como se notó en nuestro paciente. En este contexto, el estudio más grande publicado con 199 pacientes $^{(3)}$ informa niveles de TSH por debajo del límite normal en $29 \%$ de los casos. De estos, en 126 pacientes con muestras de TSH pre y posparatiroidectomía, se encontró una TSH suprimida sin sintomatología en el 15\%, TSH suprimida y síntomas leves como insomnio o un cambio en el nivel de energía en $11 \%$ de los casos y tan solo el $3 \%$ de los pacientes desarrolló una tiroiditis franca con requerimiento de tratamiento farmacológico.

Existen también reportes de casos de tiroiditis debido a lesiones penetrantes e infecciosas de la tiroides. Nishihara et al. ${ }^{(8)}$ reportaron el caso de tiroiditis debido a una complicación infecciosa secundaria a biopsia con aguja fina de tiroides en una mujer de 39 años, Kobayashi et al. ${ }^{(9)}$ ya habían reportado este tipo de complicación, luego de aspiración de quistes tiroideos, enfermedad que denominaron tiroiditis posaspiración. También se ha documentado la presencia de tiroiditis en pacientes sometidos a cirugía tiroidea o que involucre la manipulación de la misma ${ }^{(16)}$.

Se han reportado casos de tiroiditis secundarios a traumas no penetrantes a cuello, como en el caso de golpes contusos durante peleas o asaltos. Yoshida et al. ${ }^{(17)}$ reportaron el caso de una mujer de 21 años de edad que desarrolló una tiroiditis luego de ser asaltada y golpeada en la cara y en la porción an-

Tabla 2. Casos de tiroiditis postrauma

\begin{tabular}{|c|c|c|}
\hline Clase de tiroiditis & Pacientes & Autor \\
\hline \multicolumn{3}{|l|}{ Tirotoxicosis posquirúrgica } \\
\hline HPT terciario & 2 casos & Lederer $^{(4)}$ \\
\hline HPTP & 4 casos & Bergenfelz $z^{(13)}$ \\
\hline HPTP & 11 casos & Lindblom $^{(14)}$ \\
\hline HРTP & 1 caso & Musi $i^{(15)}$ \\
\hline Laringectomía & 44 casos & Salazar ${ }^{(5)}$ \\
\hline HPTP & 40 casos & Stang $^{(3)}$ \\
\hline Laringofaringoesofagectomía & 1 caso & Calle ${ }^{(16)}$ \\
\hline Laringectomía & 1 caso & Blenke $^{(21)}$ \\
\hline Laringectomía & 1 caso & McDermott ${ }^{(22)}$ \\
\hline \multicolumn{3}{|l|}{ Trauma } \\
\hline Posaspiración & 6 casos & Kobayashi(9) \\
\hline Tridente de pesca & 1 caso & Delikoukos $^{(23)}$ \\
\hline Trauma contuso & 2 casos & Delikoukos $^{(23)}$ \\
\hline Artes marciales & 1 caso & Blum $^{(2)}$ \\
\hline Estrangulación & 1 caso & Ramírez $^{(11)}$ \\
\hline Trauma & 1 caso & Kanbay ${ }^{(24)}$ \\
\hline Accidente de tránsito & 1 caso & Hagiwara ${ }^{(25)}$ \\
\hline Cinturón de seguridad & 1 caso & Leckie $^{(7)}$ \\
\hline
\end{tabular}

HPTP: hiperparatiroidismo primario. HPT: hiperparatiroidismo 
terior del cuello. Ramírez et al. ${ }^{(11)}$ también reportaron el caso de una mujer de 37 años de edad que desarrolló una tormenta tiroidea luego de ser asaltada realizándole un torniquete en el cuello. Leckie et al. ${ }^{(7)}$ reportaron el caso de una mujer de 64 años de edad que presentaba un bloqueo en la captación de ${ }^{99 \mathrm{~m}} \mathrm{Tc}$ en el lóbulo izquierdo de la tiroides, posterior a un trauma crónico en el cuello con el cinturón de seguridad de su carro. Blum et al. ${ }^{(2)}$ reportaron el caso de un practicante de Aikido que desarrolló dos episodios de tiroiditis secundaria a trauma contuso directo al cuello anterior durante la práctica de artes marciales, en quien no se encontraron hallazgos de laboratorio sugestivos de otras patologías tiroideas. Los dos episodios que tuvo fueron cortos y tres años después del seguimiento estaba clínicamente asintomático y eutiroideo.

Carney et al. $^{(10)}$ reportaron una serie de estudios con perros a quienes se realizaban palpaciones vigorosas de la tiroides, desarrollando características histopatológicas de una foliculitis granulomatosa multifocal, lesión que ellos encontraron en tiroides extraídas quirúrgicamente, bien sea por cáncer tiroideo o por cáncer laríngeo. La importancia de este hallazgo, como se sugiere, es pobre en términos de relevancia clínica. Hwang et al. ${ }^{(18)}$ aportaron al entendimiento patológico de este hallazgo, sugiriendo que la tiroiditis por palpación no es solamente secundaria a una daño mecánico de los folículos tiroideos sino también a una alteración fisiopatológica en las membranas basales de los mismos. Ante la escasez de información no se tienen reportes del comportamiento de los títulos de anticuerpos contra la tiroides que pudieran generarse en el proceso de liberación masiva del contenido glandular tiroideo.

También hay varios reportes de casos de pacientes politraumatizados o que consultan por otros traumas no direc-

\section{Referencias}

1. Meurisse M, Gollogly L, Degauque C, Fumal I, Defechereux T, Hamoir E. Iatrogenic thyrotoxicosis: causal circumstances, pathophysiology, and principles of treatment-review of the literature. World J Surg. 2000;24(11):1377-85.

2. Blum M, Schloss MF. Martial-arts thyroiditis. N Engl J Med. 1984;311(3):199200.

3. Stang MT, Yim JH, Challinor SM, Bahl S, Carty SE. Hyperthyroidism after parathyroid exploration. Surgery. 2005;138(6):1058-64; discussion 64-5.

4. Lederer SR, Schiffl H. Transient hyperthyroidism after total parathyroidectomy for tertiary hyperparathyroidism: a report of two cases.Wien Klin Wochenschr. 2008;120(13-14):432-4. doi: 10.1007/s00508-008-0996-3.

5. Salazar Thieroldt E, Boado Lama J, Molinero Abad S, Miján de la Torre A. Transient hyperthyroidism after total laryngectomy for laryngeal cancer. Nutr Hosp. 2014 Oct 24;31(n01):380-383.

6. Yoon SJ, Kim DM, Kim JU, Kim KW, Ahn CW, Cha BS, et al. A case of thyroid storm due to thyrotoxicosis factitia. Yonsei Med J. 2003;44(2):351-4.

7. Leckie RG, Buckner AB, Bornemann M. Seat belt-related thyroiditis documented with thyroid Tc-99m pertechnetate scans. Clin Nucl Med. 1992;17(11):859-60.

8. Nishihara E, Miyauchi A, Matsuzuka F, Sasaki I, Ohye H, Kubota S, et al. Acute suppurative thyroiditis after fine-needle aspiration causing thyrotoxicosis. Thyroid. 2005;15(10):1183-7.

9. Kobayashi A, Kuma K, Matsuzuka F, Hirai K, Fukata S, Sugawara M. Thyrotoxicosis after needle aspiration of thyroid cyst. J Clin Endocrinol Metab. 1992;75(1):21-4.

10. Carney JA, Moore SB, Northcutt RC, Woolner LB, Stillwell GK. Palpation thyroiditis (multifocal granulomatour folliculitis). Am J Clin Pathol. 1975;64(5):639-47.

11. Ramirez JI, Petrone P, Kuncir EJ, Asensio JA. Thyroid storm induced by strangulation. South Med J. 2004;97(6):608-10. tamente relacionados con el cuello anterior o la tiroides, en quienes se descubrió bien sea por síntomas asociados o por exámenes rutinarios la presencia de una tiroiditis. Jacobs et al. ${ }^{(19)}$ reportaron el caso de una adolescente que tuvo una fractura de fémur y síntomas leves de tirotoxicosis, encontrándose hallazgos de laboratorio compatibles con tiroiditis. Doussin et al. ${ }^{(20)}$ reportaron el caso de una niña de 15 años de edad severamente politraumatizada en un accidente automovilístico, desarrollando un síndrome de dificultad respiratoria del adulto y coagulación intravascular diseminada. La paciente estuvo en una unidad de cuidados intensivos durante cinco semanas; sin embargo, persistía taquicárdica sin otros signos de infección. El examen clínico y de laboratorio fue compatible con una tirotoxicosis por trauma.

En conclusión, se reporta un caso de tiroiditis postrauma, esta vez secundario a un intento de suicidio mediante ahorcamiento, engrosando la pequeña lista de reportes de casos de tiroiditis postrauma y contribuyendo con una revisión de los casos reportados en la literatura. Debe sospecharse esta entidad en pacientes con antecedente de trauma en el cuello por cualquier causa que curse con síntomas sugestivos de tirotoxicosis, como taquicardia, temblor, diaforesis, intolerancia al calor e incluso fiebre o hallazgos sugestivos de tormenta tiroidea.

\section{Agradecimientos}

Al grupo de Psiquiatría, Neurología Clínica, Neurocirugía, Medicina Interna, Medicina Nuclear y Cuidados Intensivos del Hospital Universitario San Vicente Fundación, por el cuidado brindado al paciente durante su estadía. A la doctora Paula Granda y al doctor Guillermo Latorre Sierra.

12. Wartofsky L. Clinical criteria for the diagnosis of thyroid storm. Thyroid 2012;22(7):659-60.

13. Bergenfelz A, Ahren B. Hyperthyroxinemia after surgery for primary hyperparathyroidism. Langenbecks Arch Chir. 1994;379(3):178-81.

14. Lindblom $P$, Valdemarsson $S$, Westerdahl J, Tennvall J, Bergenfelz A. Hyperthyroidism after surgery for primary hyperparathyroidism. Langenbecks Arch Surg. 1999;384(6):568-75.

15. Musi N, Braverman LE, Norris CM, Jr. Severe thyrotoxicosis after parathyroid surgery for hyperparathyroidism. Am J Med. 2000;108(6):519-20.

16. Calle RA, Cohen KL. Transient thyroiditis due to surgical trauma. Am J Med. 1993;95(5):546-8.

17. Yoshida D. Thyroid storm precipitated by trauma. J Emerg Med. 1996;14(6):697-701.

18. Hwang TS, Park SH. Histopathologic study of the so called 'palpation thyroiditis'. J Korean Med Sci. 1988;3(1):27-9.

19. Jacobs RR. Acute hyperthyroidism precipitated by trauma. South Med J. 1979;72(7):890-1.

20. Doussin JF, Dubost J, Banssillon V. [Post-traumatic hyperthyroxinemia or hyperthyroidism]. Ann Fr Anesth Reanim. 1985;4(1):72-4.

21. Blenke EJ, Vernham GA, Ellis G. Surgery-induced thyroiditis following laryngectomy. J Laryngol Otol. 2004;118(4):313-4.

22. McDermott A, Onyeaka CV, Macnamara M. Surgery-induced thyroiditis: fact or fiction? Ear Nose Throat J. 2002;81(6):408-10.

23. Delikoukos S, Mantzos F. Thyroid storm induced by trauma due to spear fishing-gun trident impaction in the neck. Emerg Med J. 2007;24(5):355-6.

24. Kanbay M, Sengul A, Güvener N. Trauma induced thyroid storm complicated by multiple organ failure. Chin Med J (Engl). 2005;118(11):963-5.

25. Hagiwara A, Murata A, Matsuda T, Sakaki S, Shimazaki S. Thyroid storm after blunt thyroid injury: a case report. J Trauma. 2007;63(3):E85-7. 